\title{
SOME CHARACTERIZATIONS OF $n$-DIMENSIONAL $F$-SPACES
}

\author{
BY \\ M. J. CANFELL( ${ }^{(1)}$
}

\begin{abstract}
In this paper we obtain characterizations of an $n$-dimensional $F$-space in terms of the rings of continuous real-valued and complex-valued functions defined on the space. Motivation for these results is the work of Gillman and Henriksen on $U$-spaces ( $F$-spaces of dimension 0$)$ and $T$-spaces ( $F$-spaces of dimension 0 or 1$)$.
\end{abstract}

1. Introduction. Throughout, $X$ denotes a completely regular (Hausdorff) space, $C(X)$ the ring of continuous real-valued functions on $X$, and $C^{*}(X)$ the subring of $C(X)$ consisting of the bounded functions in $C(X)$.

By definition, $X$ is an $F$-space if $C(X)$ has the property that finitely generated ideals in $C(X)$ are principal [5], [6]. Our main concern here is to define a condition on commutative rings with identity in such a way that $X$ is an $n$-dimensional $F$-space if and only if $C(X)$ satisfies this condition. The condition we select, called $H_{n}$, corresponds to condition $T$ of [4] when $n=1$. In Theorem 3, we prove that $X$ is an $n$-dimensional $F$-space if and only if $C(X)$ satisfies condition $H_{n}$. Characterizations of topological dimension alone in terms of $C(X)$ have been given in [2] and [6, Theorem 16.35].

In Theorems 3 and 4 we give characterizations of $F$-spaces and $n$-dimensional $F$-spaces in terms of the rings of continuous complex-valued functions defined on them. These characterizations are analogous to those in terms of $C(X)$ and are of interest in connection with sup-norm algebras of complex continuous functions [8], and alignable complex Banach lattices [1].

For $f \in C(X)$ we define $Z(f)=\{x \in X: f(x)=0\}$ (the zero-set of $f), P(f)$ $=\{x \in X: f(x)>0\}$ and $N(f)=\{x \in X: f(x)<0\}$. For the elementary properties of zero-sets the reader is referred to [6].

We use the modification of covering dimension involving basic covers given in [6, p. 243]. By a slight modification of Definition 4 of [3], we obtain the following characterization of dimension.

Received by the editors August 13, 1970.

AMS 1969 subject classifications. Primary 5420, 5470; Secondary 1310, 5428.

Key words and phrases. $n$-dimensional, $F$-space, Hermite ring, $H_{n}$-ring, rings of continuous functions.

(1) Some of the results presented here were included in a thesis written at the University of Edinburgh. I am greatly indebted to my supervisor Professor F. F. Bonsall for his suggestions and encouragement. 
LEMMA 1. $\operatorname{dim} X \leqq n$ if and only if given $n+1$ disjoint pairs $C_{i}, C_{i}^{\prime}, i=1, \ldots, n+1$, of zero-sets of $X$, there exist functions $k_{i} \in C(X)$ such that $k_{i}\left(C_{i}\right)=\{1\}, k_{i}\left(C_{i}^{\prime}\right)=\{-1\}$, $-1 \leqq k_{i} \leqq 1$, and $\bigcap_{i=1}^{n+1} Z\left(k_{i}\right)=\varnothing$.

Proof. Necessity. If $C_{i}$ and $C_{i}^{\prime}$ are disjoint zero-sets, we can choose $f_{i} \in C(X)$ such that $f_{i}\left(C_{i}\right)=\{1\}, f_{i}\left(C_{i}^{\prime}\right)=\{-1\}$ and $-1 \leqq f_{i} \leqq 1$. Let $I^{n+1}=[-1,1]^{n+1}$ and let $S^{n}$ denote the surface of $I^{n+1}$. Then $f=\left(f_{1}, \ldots, f_{n+1}\right)$ is a continuous mapping of $X$ into $I^{n+1}$. Since $\operatorname{dim} X \leqq n$, we can, by Definition 3 of [3], choose $k=\left(k_{1}, \ldots, k_{n+1}\right): X \rightarrow S^{n}$ such that $k(x)=f(x)$ whenever $f(x) \in S^{n}$. Then the functions $k_{i}, i=1, \ldots, n+1$, satisfy the required conditions.

Sufficiency. If functions $k_{i}$ exist as stated, then $C_{i}$ and $C_{i}^{\prime}$ are separated in $X-Z\left(k_{i}\right)$ and $\bigcap_{i=1}^{n+1} Z\left(k_{i}\right)=\varnothing$. By Definition 4 of [3], $\operatorname{dim} X \leqq n$.

We now recall some properties of $F$-rings and Hermite rings. In the following $S$ will denote a commutative ring with identity. The ideal of $S$ generated by $n$ elements $a_{1}, \ldots, a_{n}$ will be denoted by $a_{1} S+\cdots+a_{n} S$. A commutative ring $S$ with identity is called an $F$-ring if every finitely generated ideal of $S$ is principal. Thus $X$ is an $F$-space if and only if $C(X)$ is an $F$-ring.

We take the following characterization of Hermite rings [4, Lemma 4].

LEMMA 2. A commutative ring $S$ with identity is a Hermite ring if and only if it satisfies the conditions:

(i) $S$ is an F-ring.

(ii) Whenever $a_{1}, a_{2}, d \in S$ and $a_{1} S+a_{2} S=d S$, there exist $b_{1}, b_{2} \in S$ such that $a_{1}=b_{1} d, a_{2}=b_{2} d$ and $b_{1} S+b_{2} S=S$.

A completely regular space $X$ such that $C(X)$ is a Hermite ring is called a $T$-space. Alternative characterizations of $T$-spaces are given in [5, Theorem 3.2]. We will see later that $X$ is a $T$-space if and only if $X$ is an $F$-space and $\operatorname{dim} X \leqq 1$.

2 . $n$-dimensional $F$-spaces.

Definition. Let $n$ be a nonnegative integer. A commutative ring $S$ with identity is said to be an $H_{n}$-ring, or to satisfy the condition $H_{n}$, if

(i) $S$ is an $F$-ring.

(ii) Whenever $a_{1}, \ldots, a_{n+1}, d \in S$ and $a_{1} S+\cdots+a_{n+1} S=d S$, there exist $b_{1}, \ldots, b_{n+1} \in S$ such that $a_{1}=b_{1} d, \ldots, a_{n+1}=b_{n+1} d$ and $b_{1} S+\cdots+b_{n+1} S=S$.

Thus $S$ is an $H_{1}$-ring if and only if it is a Hermite ring, and $S$ is an $H_{0}$-ring if and only if it is an $F$-ring in which generators of principal ideals are unique (up to associates).

THEOREM 3. For every completely regular space $X$, the following statements are equivalent:

(a) $X$ is an $F$-space and $\operatorname{dim} X \leqq n$.

(b) $C(X)$ is an $H_{n}$-ring.

(c) $C^{*}(X)$ is an $H_{n}$-ring.

(d) For all $f_{1}, \ldots, f_{n+1} \in C(X)$, there exist $k_{1}, \ldots, k_{n+1} \in C(X)$ such that $f_{1}=k_{1}\left|f_{1}\right|, \ldots, f_{n+1}=k_{n+1}\left|f_{n+1}\right|$ and $k_{1} C(X)+\cdots+k_{n+1} C(X)=C(X)$. 
Proof. (a) $\Rightarrow\left(\right.$ d). Suppose $f_{1}, \ldots, f_{n+1} \in C(X)$. Since $X$ is an $F$-space, $P\left(f_{i}\right)$ and $N\left(f_{i}\right)$ are contained in disjoint zero-sets. By Lemma 1, there exist functions $k_{i}$ such that $k_{i}\left(P\left(f_{i}\right)\right)=\{1\}, k_{i}\left(N\left(f_{i}\right)\right)=\{-1\}$, and $\bigcap_{i=1}^{n+1} Z\left(k_{i}\right)=\varnothing$. Hence $f_{i}=k_{i}\left|f_{i}\right|$, $i=1, \ldots, n+1$, and $k_{1} C(X)+\cdots+k_{n+1} C(X)=C(X)$.

(d) $\Rightarrow$ (b). The hypothesis implies that $X$ is an $F$-space and hence that $C(X)$ is an $F$-ring. Suppose that $f_{1} C(X)+\cdots+f_{n+1} C(X)=h C(X)$. There exist $g_{1}^{\prime}, \ldots, g_{n+1}^{\prime} \in C(X)$ and $s_{1}, \ldots, s_{n+1} \in C(X)$ such that $f_{1}=g_{1}^{\prime} h, \ldots, f_{n+1}=g_{n+1}^{\prime} h$ and $h=s_{1} f_{1}+\cdots+s_{n+1} f_{n+1}=s_{1} g_{1}^{\prime} h+\cdots+s_{n+1} g_{n+1}^{\prime} h$. Put $q=1-s_{1} g_{1}^{\prime}-\cdots-$ $s_{n+1} g_{n+1}^{\prime}$.

Then $h q=0$ and for any elements $t_{i} \in C(X)$ we have $\left(g_{i}^{\prime}+t_{i} q\right) h=f_{i}$. We will choose the $t_{i}$ so that the elements $g_{i}^{\prime}+t_{i} q$ generate $C(X)$. Since $X$ is an $F$-space, there exists $p \in C(X)$ such that $p q=|q|$. By hypothesis, there exist $m_{i} \in C(X)$ such that $g_{i}^{\prime}=m_{i}\left|g_{i}^{\prime}\right|, i=1, \ldots, n+1$, and $\bigcap_{i=1}^{n+1} Z\left(m_{i}\right)=\varnothing$. Let $t_{i}=p m_{i}$ and let $g_{i}=g_{i}^{\prime}+t_{i} q$. Then for each $x \in X$, we have $g_{i}(x) \neq 0$ for some $i$. To see this, suppose first that $g_{i}^{\prime}(x) \neq 0$ for some $i$. Now $\left(t_{i} q\right)(x)=p(x) m_{i}(x) q(x)=m_{i}(x)|q(x)|$ has the same sign (or argument) as $g_{i}^{\prime}(x)$ so that $g_{i}(x) \neq 0$. On the other hand, if $g_{i}^{\prime}(x)=0$ for all $i$, then $q(x)=1, p(x)=1$, and $g_{i}(x)=t_{i}(x)=m_{i}(x)$. Since $\bigcap_{i=1}^{n+1} Z\left(m_{i}\right)=\varnothing$, then $g_{i}(x) \neq 0$ for some $i$. Hence $g_{1} C(X)+\cdots+g_{n+1} C(X)=C(X)$.

(b) $\Rightarrow($ a). By hypothesis, $C(X)$ is an $F$-ring and hence $X$ is an $F$-space. Suppose that $C_{i}, C_{i}^{\prime}, i=1, \ldots, n+1$, are $n+1$ disjoint pairs of zero-sets. Choose $f_{i} \in C(X)$ such that $f_{i}\left(C_{i}\right)=\{1\}, f_{i}\left(C_{i}^{\prime}\right)=\{-1\}$, for $i=1, \ldots, n+1$, and let $h=\left|f_{1}\right|+\cdots+\left|f_{n+1}\right|$. Since $X$ is an $F$-space, $f_{1} C(X)+\cdots+f_{n+1} C(X)=h C(X)$. By hypothesis, there exist $g_{i} \in C(X)$ such that $f_{i}=g_{i} h$ and $g_{1} C(X)+\cdots+g_{n+1} C(X)=C(X)$. Thus $\bigcap_{i=1}^{n+1} Z\left(g_{i}\right)=\varnothing$. Now $P\left(f_{i}\right) \subset P\left(g_{i}\right), N\left(f_{i}\right) \subset N\left(g_{i}\right)$ for $i=1, \ldots, n+1$. Also $\left|g_{i}(x)\right| \leqq 1$ for $f_{i}(x) \neq 0$ and we can arrange that $\left|g_{i}(x)\right| \leqq 1$ everywhere (take $g_{i}^{\prime}(x)=g_{i}(x)$ if $\left|g_{i}(x)\right| \leqq 1$ and $g_{i}^{\prime}(x)=g_{i}(x) /\left|g_{i}(x)\right|$ if $\left.\left|g_{i}(x)\right| \geqq 1\right)$. Since $P\left(g_{i}\right)$ and $N\left(g_{i}\right)$ are completely separated, we can choose $s_{i}$ so that $s_{i}\left(P\left(g_{i}\right)\right)=\{1\}$ and $s_{i}\left(N\left(g_{i}\right)\right)=\{0\}$.

Let $m_{i} \in C(X)$ satisfy $f_{i}=m_{i}\left|f_{i}\right|, \quad-1 \leqq m_{i} \leqq 1$. Define $k_{i}=s_{i} \max \left\{m_{i}, g_{i}\right\}+$ $\left(1-s_{i}\right) \min \left\{m_{i}, g_{i}\right\}$. Then $f_{i}=k_{i}\left|f_{i}\right|$ and $Z\left(k_{i}\right) \subset Z\left(g_{i}\right)$. Hence $\bigcap_{i=1}^{n+1} Z\left(k_{i}\right)=\varnothing$. Since $k_{i}\left(C_{i}\right)=\{1\}$ and $k_{i}\left(C_{i}^{\prime}\right)=\{-1\}$ we have $\operatorname{dim} X \leqq n$ by Lemma 1 .

(b) $\Leftrightarrow(\mathrm{c}) . C^{*}(X)$ is isomorphic to $C(\beta X)$ where $\beta X$ is the Stone-Čech compactification of $X$. Since $\operatorname{dim} X=\operatorname{dim} \beta X[6$, p. 245] and $X$ is an $F$-space if and only if $\beta X$ is an $F$-space, the result follows from (a) $\Leftrightarrow$ (b) above.

EXAMPLE. $\beta R^{n}-R^{n}$ is an $n$-dimensional $F$-space.

That $\beta R^{n}-R^{n}$ is an $F$-space follows from Theorem 14.27 of [6], and it is shown in [7] that $\operatorname{dim}\left(\beta R^{n}-R^{n}\right)=n$.

As a simple consequence, we have an example of an $F$-ring which is not a Hermite ring (the first example of this was given in [5]). $\beta R^{2}-R^{2}$ is an $F$-space which is not a $T$-space, hence $C\left(\beta R^{2}-R^{2}\right)$ is an $F$-ring which is not a Hermite ring.

3. Continuous complex functions on $F$-spaces. We turn now to the problem of characterizing $F$-spaces in terms of the ring $C_{C}(X)$ of all continuous complex- 
valued functions on $X$. We also consider $C_{C}^{*}(X)$, the subring of $C_{C}(X)$ consisting of the bounded functions in $C_{C}(X)$.

Since $Z(f)=Z(|f|)$, the family of zero-sets of $C_{C}(X)$ is the same as the family of zero-sets of $C(X)$.

An ideal $I$ of $C_{C}(X)$ is said to be selfadjoint if and only if $f \in I \Rightarrow \bar{f} \in I$, where $\bar{f}$ is the complex conjugate of $f$.

THEOREM 4. The following conditions are equivalent:

(a) $X$ is an F-space.

(b) $C_{C}(X)$ is an F-ring.

(c) $C_{C}^{*}(X)$ is an F-ring.

(d) Each ideal I of $C_{C}(X)$ is selfadjoint.

(e) For all $f, g \in C_{C}(X), f C_{C}(X)+g C_{C}(X)=(|f|+|g|) C_{C}(X)$.

(f) Given a zero-set $Z$ of $X$, every function $\theta \in C_{C}^{*}(X-Z)$ has a continuous extension $h \in C_{C}^{*}(X)$.

(g) Given $f \in C_{C}(X)$, there exist $k_{1}, k_{2} \in C_{C}(X)$ such that $f=k_{1}|f|$ and $|f|=k_{2} f$.

Proof. (g) $\Rightarrow(\mathrm{d})$. Let $f \in I$. There exist $k_{1}, k_{2} \in C_{C}(X)$ such that $\bar{f}=k_{1}|\bar{f}|=k_{1}|f|$ and $|f|=k_{2} f$. Hence $\bar{f}=k_{1} k_{2} f$ so that $\bar{f} \in I$.

(d) $\Rightarrow$ (a). Let $f \in C(X)$. Then $f-i|f| \in C_{C}(X)$ and by hypothesis its complex conjugate $f+i|f|$ is in the principal ideal generated by $f-i|f|$. There exists $h \in C_{C}(X)$ such that $f+i|f|=h(f-i|f|)$. On multiplying both sides by $f-i|f|$ we have

$$
f^{2}+|f|^{2}=h\left(f^{2}-2 i|f| f-|f|^{2}\right),
$$

and on simplifying and equating real parts, we get

$$
|f|^{2}=f^{2}=I(h) f|f| \text {. }
$$

It follows that $f=I(h)|f|$ so that $X$ is an $F$-space.

The rest of the proof is a routine modification of the proofs in Theorem 14.25 of [6]. For example, (a) $\Rightarrow$ (f) since the real and imaginary parts of $\theta$ can be extended over $X$.

Although $C_{C}(X)$ is an $F$-ring if and only if $C(X)$ is an $F$-ring, the situation is slightly different for $H_{n}$-rings.

THEOREM 5. The following conditions are equivalent:

(a) $C_{C}(X)$ is an $H_{n}$-ring.

(b) $C(X)$ is an $H_{2 n+1}$-ring.

(c) $X$ is an $F$-space and $\operatorname{dim} X \leqq 2 n+1$.

(d) For all $f_{1}, \ldots, f_{n+1} \in C_{C}(X)$, there exist $k_{1}, \ldots, k_{n+1} \in C_{C}(X)$ such that $f_{1}=k_{1}\left|f_{1}\right|, \ldots, f_{n+1}=k_{n+1}\left|f_{n+1}\right|$ and $k_{1} C_{C}(X)+\cdots+k_{n+1} C_{C}(X)=C_{C}(X)$.

Proof. (a) $\Rightarrow$ (b). First we observe that if $X$ is an $F$-space and $f_{1}, f_{2} \in C(X)$, then $f_{1} C(X)+f_{2} C(X)=\left(f_{1}^{2}+f_{2}^{2}\right)^{1 / 2} C(X)$. In fact, since $\left(f_{1}^{2}+f_{2}^{2}\right)^{1 / 2} \leqq\left|f_{1}\right|+\left|f_{2}\right|$ 
$\leqq 2\left(f_{1}^{2}+f_{2}^{2}\right)^{1 / 2}$, then it follows from Theorem 14.25(6) of [6], that $\left|f_{1}\right|+\left|f_{2}\right|$ and $\left(f_{1}^{2}+f_{2}^{2}\right)^{1 / 2}$ are multiples of each other. Similarly if $f_{1}, f_{2} \in C_{C}(X)$, then $f_{1} C_{C}(X)$ $+f_{2} C_{C}(X)=\left(\left|f_{1}\right|^{2}+\left|f_{2}\right|^{2}\right)^{1 / 2} C_{C}(X)$.

Now suppose that $f_{1}, \ldots, f_{2 n+2}, d \in C(X)$ and $f_{1} C(X)+\cdots+f_{2 n+2} C(X)$ $=d C(X)$. Let $h=\left(f_{1}^{2}+\cdots+f_{2 n+2}^{2}\right)^{1 / 2}$. By hypothesis and Theorem 4, $X$ is an $F$-space and, by the preceding remarks, $d C(X)=h C(X)$. Let $g_{i}=f_{2 i-1}+i f_{2 i}$, $i=1, \ldots, n+1$. Again by the preceding remarks, $g_{1} C_{C}(X)+\cdots+g_{n+1} C_{C}(X)$ $=\left(\left|g_{1}\right|^{2}+\cdots+\left|g_{n+1}\right|^{2}\right)^{1 / 2} C_{C}(X)=h C_{C}(X)$. Therefore $g_{1} C_{C}(X)+\cdots+g_{n+1} C_{C}(X)$ $=d C_{C}(X)$. By hypothesis, there exist elements $s_{2 i-1}+i s_{2 i} \in C_{C}(X)$ which generate $C_{C}(X)$ and which satisfy $g_{i}=f_{2 i-1}+i f_{2 i}=\left(s_{2 i-1}+i s_{2 i}\right) d$. Thus $f_{i}=s_{i} d, i=1, \ldots, 2 n+2$ and $\bigcap_{i=1}^{2 n+2} Z\left(s_{i}\right)=\varnothing$, i.e., $s_{1} C(X)+\cdots+s_{2 n+2} C(X)=C(X)$.

(b) $\Rightarrow$ (c). This has been shown in Theorem 2 .

(c) $\Rightarrow$ (d). Let $f_{1}, \ldots, f_{n+1} \in C_{C}(X)$. By Theorem 4 , there exist $k_{1}^{\prime}, \ldots, k_{n+1}^{\prime}$ $\in C_{C}(X)$ such that $f_{1}=k_{1}^{\prime}\left|f_{1}\right|, \ldots, f_{n+1}=k_{n+1}^{\prime}\left|f_{n+1}\right|$. If $f_{i}(x) \neq 0$, then $\left|k_{i}^{\prime}(x)\right|=1$, and we may assume that $\left|k_{i}^{\prime}(x)\right| \leqq 1$ for $x \in X, i=1, \ldots, n+1$.

Let $D$ be the closed unit disc in the complex plane and $D_{1}$ its surface; that is, $D=\{z \in C:|z| \leqq 1\}$ and $D_{1}=\{z \in C:|z|=1\}$. Then $k^{\prime}=\left(k_{1}^{\prime}, \ldots, k_{n+1}^{\prime}\right)$ is a continuous mapping of $X$ into $D^{n+1} \subset R^{2 n+2}$. Since $\operatorname{dim} X \leqq 2 n+1$, we may, as in Definition 3 of [3], choose $k=\left(k_{1}, \ldots, k_{n+1}\right): X \rightarrow D_{1}^{n+1}$ such that $k(x)=k^{\prime}(x)$ whenever $k^{\prime}(x) \in D_{1}^{n+1}$. Thus $f_{i}=k_{i}\left|f_{i}\right|, i=1, \ldots, n+1$, and $\bigcap_{i=1}^{n+1} Z\left(k_{i}\right)=\varnothing$.

(d) $\Rightarrow$ (a). The proof is identical with (d) $\Rightarrow$ (b) of Theorem 3 .

COROLlaRY. $X$ is a T-space if and only if given $f \in C_{C}(X)$, there exists $k \in C_{C}(X)$ such that $f=k|f|$ and $Z(k)=\varnothing$.

Proof. This is (d) $\Leftrightarrow$ (b) above with $n=0$ but we give a simple direct proof. If $X$ is an $F$-space and $f=f_{1}+i f_{2}, k=k_{1}+i k_{2}$, then $f=k|f|$ and $Z(k)=\varnothing$ if and only if $f_{1}=k_{1}\left(f_{1}^{2}+f_{2}^{2}\right)^{1 / 2}, f_{2}=k_{2}\left(f_{1}^{2}+f_{2}^{2}\right)^{1 / 2}$ and $Z\left(k_{1}\right) \cap Z\left(k_{2}\right)=\varnothing$. Since $f_{1} C(X)$ $+f_{2} C(X)=\left(f_{1}^{2}+f_{2}^{2}\right)^{1 / 2} C(X)$, then (b) $\Rightarrow(\mathrm{d})$ is immediate, while (d) $\Rightarrow(\mathrm{b})$ follows from Lemma 4 of [4].

As the example $X=\beta R^{2}-R^{2}$ shows, $C_{C}(X)$ may be a Hermite ring while $C(X)$ is not a Hermite ring.

4. $U$-spaces and $T$-spaces. An element $u$ of $C(X)$ (or $C_{C}(X)$ ) is said to be unitary if $|u(x)|=1$ for all $x \in X$. If $f=v|f|$ and $Z(v)=\varnothing$, then $u=v /|v|$ is unitary, and since $|f|=|v||f|$, we have $f=v|f|=u|v||f|=u|f|$.

From Theorem 3 and the corollary to Theorem 5, we have the following characterization.

LEMMA 6. $X$ is a $U$-space (respectively $T$-space) if and only if for each $f \in C(X)$ (respectively $C_{C}(X)$ ), there exists a unitary element $u$ of $C(X)$, (respectively $C_{C}(X)$ ) such that $f=u|f|$.

Finally we given an unpublished result of Bonsall in which $T$-spaces are characterized in terms of linear operators on the complex vector space $C_{C}(X)$. 
A rotation on $C_{C}(X)$ is a linear operator $D$ mapping $C_{C}(X)$ onto $C_{C}(X)$ such that $|D f|=|f|$ for all $f \in C_{C}(X) . C_{C}(X)$ is said to be alignable if and only if given $f_{0} \in C_{C}(X)$ there exists a rotation $D$ on $C_{C}(X)$ such that $D\left|f_{0}\right|=f_{0}$.

Alignable spaces were considered in [1].

THEOREM 7. $X$ is a T-space if and only if $C_{C}(X)$ is alignable.

Proof. If $u \in C_{C}(X)$ is a unitary element for which $f_{0}=u\left|f_{0}\right|$, then clearly the operation of multiplication by $u$ is a rotation on $C_{C}(X)$ with the required property.

Conversely, suppose that $D$ is a rotation on $C_{C}(X)$ for which $D\left|f_{0}\right|=f_{0}$. We show that $D 1$ is unitary and that $D$ is the operation of multiplication by $D 1$. Given $x \in X$, let $\Psi_{x}$ and $\Phi_{x}$ denote the linear functionals on $C_{C}(X)$ defined by $\Psi_{x}(f)$ $=f(x)$ and $\Phi_{x}(f)=(D f)(x)$. Then $\left|\Psi_{x}(f)\right|=\left|\Phi_{x}(f)\right|$ for each $f \in C_{C}(X)$. Hence $\Psi_{x}$ and $\Phi_{x}$ have the same null space and therefore differ only by a scalar factor. Thus $\Phi_{x}=\lambda_{x} \Psi_{x}$ for some $\lambda_{x} \in C$ with $\left|\lambda_{x}\right|=1$. Now $(D f)(x)=\Phi_{x}(f)=\lambda_{x} \Psi_{x}(f)$ $=\lambda_{x} f(x)$. In particular, $(D 1)(x)=\lambda_{x}$ so that $(D f)(x)=((D 1)(x)) f(x)$. This holds for all $x \in X$, so that $D f=(D 1) f$. Finally, for each $x \in X,|(D 1)(x)|=\left|\lambda_{x}\right|=1$ so that $D 1$ is unitary and $(D 1)\left|f_{0}\right|=D\left|f_{0}\right|=f_{0}$.

\section{REFERENCES}

1. F. F. Bonsall and B. J. Tomuik, The semi-algebra generated by a compact linear operator, Proc. Edinburgh Math. Soc. (2) 14 (1964/65), 177-196. MR 32 \#1557.

2. M. J. Canfell, Uniqueness of generators of principal ideals in rings of continuous functions, Proc. Amer. Math. Soc. 26 (1970), 571-573.

3. J. R. Gard and R. D. Johnson, Four-dimension equivalences, Canad. J. Math. 20 (1968), 48-50. MR 36 \#5913.

4. L. Gillman and M. Henriksen, Some remarks about elementary divisor rings, Trans. Amer. Math. Soc. 82 (1956), 362-365. MR 18, 9.

5. - Rings of continuous functions in which every finitely generated ideal is principal, Trans. Amer. Math. Soc. 82 (1956), 366-391. MR 18, 9.

6. L. Gillman and M. Jerison, Rings of continuous functions, University Series in Higher Math., Van Nostrand, Princeton, N. J., 1960. MR 22 \#6994.

7. M. Jerison, Rings of germs of continuous functions, Proc. Conf. Functional Analysis (Irvine, Calif., 1966), Academic Press, London; Thompson Book, Washington, D.C., 1967. MR 36 \#4342.

8. M. L. Weiss, Some separation properties in sup-norm algebras of continuous functions, Proc. Internat. Sympos. Function Algebras (Tulane University, 1965), Scott-Foresman, Chicago, Ill., 1966, pp. 93-97. MR 33 \#1756.

UNIVERSITY OF NEW ENGLAND, Armidale, Australia 